\title{
Automation of the Czech part of the European fireball network: equipment, methods and first results
}

\author{
Pavel Spurný, Jiří Borovička and Lukáš Shrbený \\ Astronomical Institute of the Academy of Sciences, 25165 Ondřejov, Czech Republic \\ email: spurny@asu.cas.cz
}

\begin{abstract}
In the last several years the manually operated fish-eye cameras in the Czech part of the European fireball Network (EN) have been gradually replaced with new generation cameras, the modern and sophisticated completely autonomous fireball observatories (AFO), which were recently developed in the Czech Republic. The main motivation for construction of this new observing system was to continue in regular fireball observations and to make these observations more complex and efficient. In this paper we briefly describe basic design and work of this new instrument and its deployment at the Czech stations of the EN. The current dislocation of the individual stations and their equipment is also discussed. Along with this new modern instrument we developed also new software for measurement of photographic negatives which makes this time consuming work more efficient and easier. The AFOs provide us with data on fireballs far richer and more interesting than those we were able to get in the past. This is illustrated by the cases of two recently observed fireballs which were recorded by the AFOs. We describe the high precision of all the measureded values as well as the very detailed information about light curves in both cases.
\end{abstract}

Keywords. Meteors; meteoroids; instrumentation

\section{Introduction}

The Earth steadily interacts with meteoroids - small interplanetary bodies of various dimensions, masses, composition and structure. A part of meteoroids are clearly linked to comets as shower meteors and another part is linked to asteroids and represents the densest part of the interplanetary matter. All meteorites with known pre-atmospheric orbits have the origin in the main belt of asteroids. The penetration of larger meteoroids through the atmosphere gives rise to spectacular luminous events - fireballs. Their photographic recordings provide excellent means to examine physical properties as well as the temporal and spatial distribution of extraterrestrial matter in the near-Earth space. The most efficient tools for registration of these very scarce events are the fireball networks, systems covering large areas of the Earth's surface, with multiple camera stations designed to image a large fraction of the night sky. The first such camera network has been established in the Czech Republic and without interruption has been in regular operation until now.

\section{Basic instrument}

In the last several years the manually operated fish-eye cameras in the Czech part of the European Fireball Network (EN) have been gradually replaced with new generation cameras, modern and sophisticated completely autonomous fireball observatories. The 


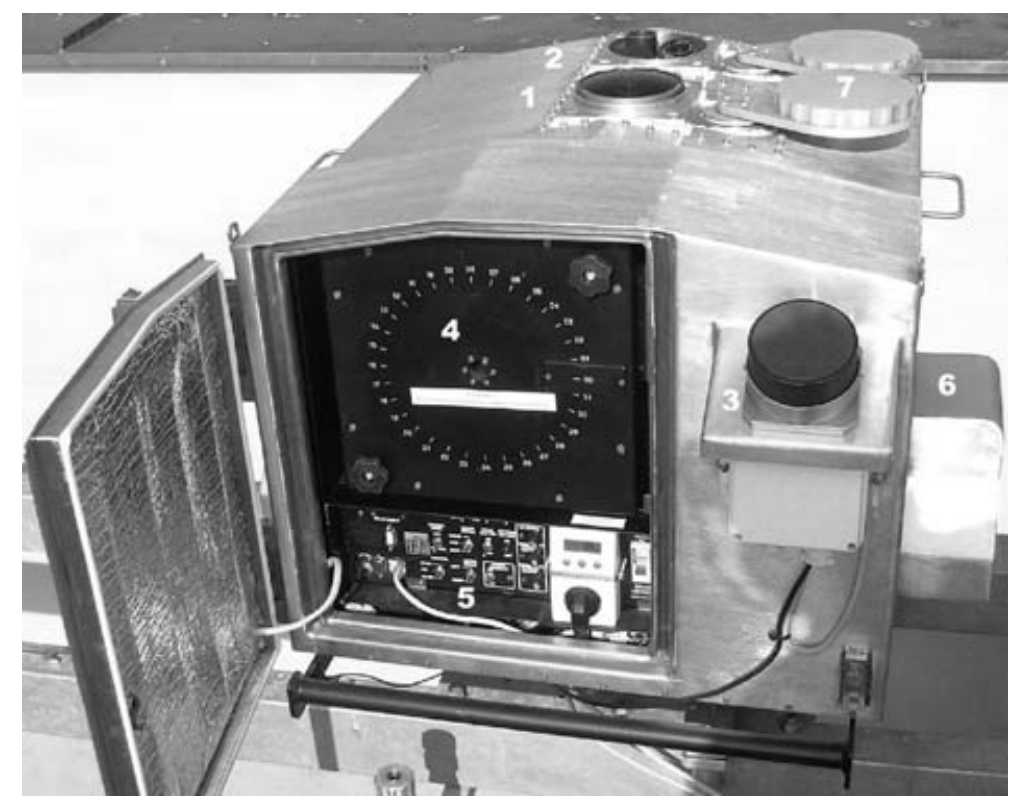

Figure 1. The Autonomous Fireball Observatory. 1- fish-eye lens; 2-battery of sensors (for cloudiness, sky brightness and sound); 3 - precipitation detector; 4-rotating cartridge with films; 5 -control panel; 6 -fans for cooling; 7 -covers.

main motivation for the construction of this new observing system was to continue in regular fireball observations and to make these observations more complex and efficient.

\subsection{Autonomous Fireball Observatory (AFO)-description of design and work}

The main function of the Autonomous Fireball Observatory is the same as for manually operated cameras: to take photographic images of the whole sky with good resolution. The AFOs main lens is the very precise Zeiss Distagon 3.5/30 $\mathrm{mm}$ fish-eye lens, and we use large format panchromatic sheet films $(9 \times 12 \mathrm{~cm}$, Ilford FP4), the same used in the old manually operated cameras. The diameter of the sky on the image is $8 \mathrm{~cm}$ and an angular resolution of 1 arc minute over the whole sky is reached on the average. We initially thought of the use of CCD sensors, however due to specific requirements for fireball registrations, such as all-night continuous recording, very wide dynamic range of recorded events $(\sim 15$ stellar magnitudes) and high angular resolution, we found that the photographic emulsion is still the most suitable for this purpose.

The observatory is shown in Fig. 1. The mass of the whole unit is about $120 \mathrm{~kg}$ and the dimensions are $80 \times 60 \times 60 \mathrm{~cm}$. The observatory is weather resistant and sealed against dust, guaranteeing reliable operations also in remote areas with hostile climatic conditions. Lens and camera interior are maintained at constant temperature. The camera is designed to use minimal electrical power and short outages of the electricity distribution network are taken care of by a back-up power supply.

The back-up supply ensures that the camera is safely put into sleep mode, where it stands by for reinstatement of the electricity supply. The camera takes exposure automatically according to a prescribed schedule corrected by actual weather conditions, which are recognized directly by the camera. Each camera unit contains a magazine of 32 films. Human intervention is thus limited to the replacement of the magazine every 5-7 weeks, depending on the number of clear nights. The camera contains a simple weather station that automatically evaluates climatic conditions before and during the 


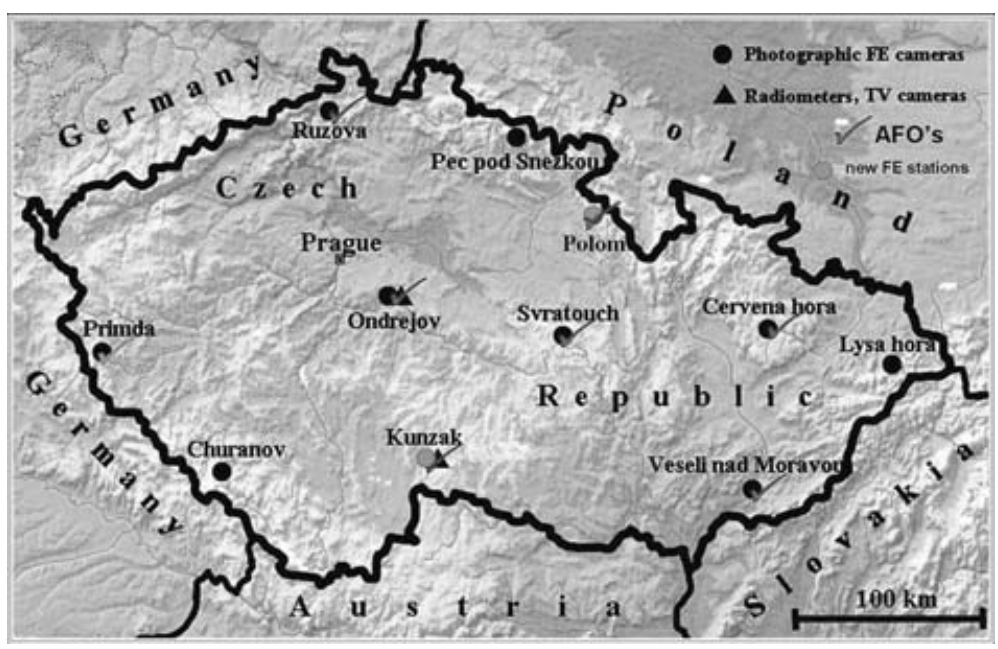

Figure 2. Map of the Czech Fireball Network.

exposure. The exposure is started and proceeds under favorable conditions only, that is when no precipitation is detected and the sky is sufficiently clear. Cloudiness is detected by a very sensitive CCD camera. This information is digitally processed and continuously evaluated. The result of this evaluation is the number of stars in the observed section of sky, which is compared with a specified limit. The film is held exactly flat by underpressure from a vacuum pump. A two-blade rotating shutter with remotely selectable rotation speed is located just above the film. This allows the speed of the fireball to be determined. The unit receives absolute time/date signals from a public internet server. The times of the beginning and end of the exposure are written to a hard disk, and an exposure identification number printed on the film. If a film is exposed it is exchanged at the end of the night and protective covers are closed over the objective and the sensors. Because only one film is used each clear night we need another method to record the time of a fireball. The camera therefore includes a photoelectric brightness sensor which monitors the total brightness of the sky continuously during the night when no precipitation is detected. The brightness level and time are recorded with a frequency of 500 measurements per second. Therefore, along with precise time information we obtain also very detailed light curves. After long tests we know that these detectors are so sensitive that we have such information for all fireballs bright enough to be recorded photographically. This radiometer is accompanied by a sound detector, which is activated by sufficiently bright and long light pulses and records the sound for up to 10 minutes. In the case of a bright bolide passage, the camera distinguishes it and automatically informs us by email or SMS message. This fact very nicely documents the high technical level of this very modern instrument, which is completely a Czech product. The cameras are networked with internet connection. Each night, the camera logs every operation and these files along with exposure diaries and event reporting are transferred to the central server at Ondřejov.

The AFOs provide us with more complex data on fireballs than what we got before with older equipment. This instrument was designed and developed by the Czech company Space Devices Ltd. with close cooperation with the Astronomical Institute of the Czech Academy of Sciences which has funded this entire project. The Space Devices company is able to produce large numbers of AFOs. 
Table 1. Positions of the stations of the Czech Fireball Network.

\begin{tabular}{|cl|c|c|c|}
\hline \multirow{2}{*}{ Number and Name } & \multicolumn{3}{|c|}{ WGS84 System } \\
\cline { 3 - 5 } & $\lambda\left({ }^{\circ} E\right)$ & $\varphi\left({ }^{\circ} E\right)$ & $h(m)$ \\
\hline 2 & Kunžak & 15.200930 & 49.107290 & 656.1 \\
3 & Rǔžová & 14.286526 & 50.834114 & 348.5 \\
4 & Churáňov & 13.614948 & 49.068430 & 1119.2 \\
9 & Svratouch & 16.033917 & 49.735111 & 736.0 \\
10 & Polom & 16.322249 & 50.350154 & 748.2 \\
11 & Přimda & 12.677897 & 49.669358 & 745.0 \\
12 & Veselí nad Moravou & 17.369622 & 48.954122 & 176.2 \\
14 & Červená hora & 17.541962 & 49.777261 & 749.7 \\
16 & Lysá hora & 18.447643 & 49.546408 & 1324.4 \\
17 & Pec pod Sněžkou & 15.729276 & 50.692143 & 823.1 \\
20 & Ondřejov & 14.780042 & 49.910128 & 525.0 \\
\hline
\end{tabular}

\subsection{Current status of the Czech Fireball Network and AFOs deployment in the Czech Republic}

The Czech Fireball Network, the founding part of the European Fireball Network, started regular operation in the fall of 1963, and has remained in full operation until the present time. This network has undergone important changes in the past four decades in terms of geographical coverage, camera equipment and management. The last important change and improvement of the network operation is the replacement of manual all-sky cameras with the new automated fireball observatories described in the previous subsection. The big advantage of the new AFO consists in its completely autonomous work. It does not need every-night human intervention and service which was the most important limiting factor for reliable work of each particular station. In terms of service, the AFO needs only a site with electric power and internet connection. Other requirements for good location of fireball station, i.e. sufficiently dark sky and good horizon remain the same. Most of our stations satisfy these needs and have stayed at the same locations, but some of them were replaced. Since December 2003 we have built two completely new stations - 10 Polom and 2 Kunžak, and replaced old manual all-sky cameras with the AFOs at stations 12 Veselí nad Moravou, 9 Svratouch, 3 Růžová, 14 Červená hora and 11 Přimda. One AFO is also in regular operation at the central station of the Czech Fireball Network, at Ondřejov Observatory along with other instruments for fireball observations which are still operated manually. The current dislocation of the fireball stations over the Czech Republic is shown in Figure 2. At present time we operate 11 stations and 8 of them are equipped with the new AFOs. The remaining two mountain stations Churáñov and Lysá hora will be rebuilt for automated operation in 2007 and the last station Pec pod Snežkou, which is close to a quickly developing tourist center, will be gradually closed because of unsatisfactory observing conditions. The list of present stations with their geographic coordinates is given in Table 1.

For completeness, we still operate two additional all-sky cameras (with the same lens) in a driven regime at the Ondřejov Observatory and Churáňov station. The main purpose of operation of these two guided cameras was to obtain the time of the fireball passage from simultaneous fixed and guided photographic records. However, the AFOs provide us this information more precisely and therefore the operation of guided cameras will be reduced in near future. 

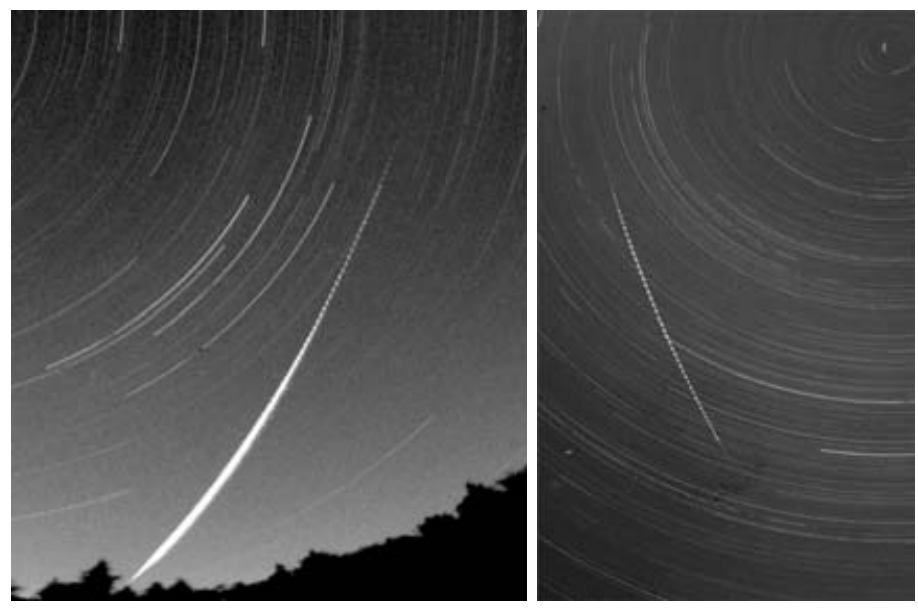

Figure 3. Parts of the all-sky photographs. The left one displays the EN280506 fireball as recorded at Ondřejov observatory, the right one the EN300706 fireball as recorded at Polom.

Along with the all-sky cameras, we operate also a battery of six long focus horizontal cameras at Ondřejov Observatory with objectives Tessar $(1 / 3.5, f=360 \mathrm{~mm})$. These cameras cover a part of the sky where we have very good horizon (covering about 230 degrees in azimuth and 30 degrees in elevation) to get information on precise position of either deep penetrating fireballs close to Ondřejov or very distant events.

To obtain more complex information on some suitably located and bright events, the photographs of fireballs in the direct light are accompanied by the spectral records from a battery of another six cameras at Ondřejov observatory equipped with similar type of lenses as the horizontal ones.

\section{Methods}

After the photographic films are developed, they are searched visually for meteors. Negatives containing meteors recorded at least at two stations are measured. The classical way of measurement makes use of the Ascorecord measuring device. This provides excellent positional accuracy but is time consuming. The operator has to identify the star trails and meteor points and adjust their positions manually. In order to compute meteor brightness, the widths of star trails and the meteor trail are to be measured. To make the process quicker and easier, we have developed a new software, called Fishscan, which enables the measurement to be done on PC using scanned negatives. After entering the coordinates of the station and the exposure times, star trails are identified semi-automatically. Positional measurement is done simply by clicking on the beginnings or ends of selected star trails, on some points along the meteor trail, and on the positions of shutter breaks along the meteor trail (for the velocity measurement). Brightness measurements are realized by making photometric scans across the star trails and across the meteor trail (between the shutter breaks) and by integrating the signal along the scans.

We have made extensive tests of the accuracy of the new method. The positional accuracy critically depends on the precision of the scanner used to convert the photographic negative into digital form. No commercial scanner was found to give a precision comparable to the manual Ascorecord measurement. Systematic positional errors were present across the negative in all cases. Only a professional photogrammetric scanner gave results equally good as the Ascorecord method. On the other hand, even cheap scanners yielded 
Table 2. Atmospheric trajectory data of the EN280506 and EN300706 fireballs.

\begin{tabular}{lc|r|r}
\hline Fireball No. & & EN280506 & \multicolumn{1}{c}{ EN300706 } \\
\hline Date & & 28.5 .2006 & 30.7 .2006 \\
Time & $(\mathrm{UT})$ & $23^{h} 16^{m} 42.0^{s} \pm 0.1^{s}$ & $20^{h} 23^{m} 21.3^{s} \pm 0.1^{s}$ \\
Maximum abs. magnitude & & -11.4 & -7.3 \\
Initial mass & $(\mathrm{kg})$ & 65. & 2.3 \\
Beginning height & $(\mathrm{km})$ & $88.04 \pm 0.02$ & $83.424 \pm 0.014$ \\
Beginning longitude & $(\mathrm{deg} \mathrm{E})$ & $15.8335 \pm 0.0003$ & $16.6433 \pm 0.0003$ \\
Beginning latitude & $(\mathrm{deg} \mathrm{N})$ & $50.43274 \pm 0.00011$ & $50.09692 \pm 0.00012$ \\
Terminal height & $(\mathrm{km})$ & $30.50 \pm 0.02$ & $37.020 \pm 0.007$ \\
Terminal longitude & $(\mathrm{deg} \mathrm{E})$ & $16.0474 \pm 0.0003$ & $16.73842 \pm 0.00013$ \\
Terminal latitude & $(\mathrm{deg} \mathrm{N})$ & $51.25030 \pm 0.00011$ & $50.54122 \pm 0.00006$ \\
Length of trajectory & $(\mathrm{km})$ & 109.41 & 68.48 \\
Duration & $(\mathrm{s})$ & 6.79 & 5.01 \\
Slope & $(\mathrm{deg})$ & 31.32 & 42.44 \\
PE/Type & & $-4.59 / \mathrm{I}$ & $-4.58 / \mathrm{I}$ \\
Stations used for computation & $10,3,20,14$ & $10,14,9,20$ \\
\hline
\end{tabular}

Table 3. Radiants and orbital elements (J2000.0) of the EN280506 and EN300706 fireballs.

\begin{tabular}{lc|r|r}
\hline Fireball No. & & \multicolumn{1}{|c}{ EN280506 } & \multicolumn{1}{|c}{ EN300706 } \\
\hline RA of apparent radiant & $(\mathrm{deg})$ & $243.324 \pm 0.013$ & $265.034 \pm 0.014$ \\
DE of apparent radiant & $(\mathrm{deg})$ & $-6.99 \pm 0.02$ & $3.235 \pm 0.019$ \\
Initial velocity & $(\mathrm{km} / \mathrm{s})$ & $17.576 \pm 0.005$ & $15.964 \pm 0.002$ \\
RA of geocentric radiant & $(\mathrm{deg})$ & $241.369 \pm 0.015$ & $263.063 \pm 0.016$ \\
DE of geocentric radiant & $(\mathrm{deg})$ & $-14.99 \pm 0.02$ & $-4.95 \pm 0.02$ \\
Geocentric velocity & $(\mathrm{km} / \mathrm{s})$ & $13.650 \pm 0.007$ & $11.480 \pm 0.003$ \\
Heliocentric velocity & $(\mathrm{km} / \mathrm{s})$ & $33.377 \pm 0.004$ & $38.028 \pm 0.003$ \\
Semimajor axis & $(\mathrm{AU})$ & $1.3936 \pm 0.0006$ & $2.942 \pm 0.002$ \\
Eccentricity & & $0.4691 \pm 0.0003$ & $0.6737 \pm 0.0002$ \\
Perihelion distance & $(\mathrm{AU})$ & $0.73983 \pm 0.00015$ & $0.95979 \pm 0.00004$ \\
Aphelion distance & $(\mathrm{AU})$ & $2.0474 \pm 0.0013$ & $4.924 \pm 0.004$ \\
Argument of perihelion & $(\mathrm{deg})$ & $261.10 \pm 0.03$ & $210.173 \pm 0.013$ \\
Ascending node & $(\mathrm{deg})$ & $67.41320 \pm 0.00007$ & $127.45038 \pm 0.00001$ \\
Inclination & $(\mathrm{deg})$ & $2.550 \pm 0.010$ & $5.566 \pm 0.007$ \\
\hline
\end{tabular}

meteor photometry more accurate than the Ascorecord method based on trail widths (see Section 4.2).

Computation of meteor trajectories, orbits and other data analysis is done by the procedures developed at the Ondřejov Observatory in the last decades. Positional reduction of all-sky images is performed by the REDSKY procedure of Borovička et al. (1995). Meteor trajectories and orbits are computed by the methods described by Ceplecha (1987). The method of Borovička (1990) is used to check the consistency of results. The approach of Ceplecha et al. (1993) is applied to study meteor dynamics, ablation and fragmentation. If a meteorite fall is suspected, the meteorite landing area is computed by the method described in Ceplecha (1987). The camera system achieves usually an absolute positional accuracy of 10-20 m for each point on a fireball's luminous trajectory (for a body at distances up to $150 \mathrm{~km}$ from the camera), and a dynamic precision of the order of $10 \mathrm{~m} / \mathrm{s}$. The precision decreases with increasing distance but we can detect fireballs and reliably determine all important values up to distances of 300-400 km from the stations. In some particular cases we can get reliable results from distances even larger than $500 \mathrm{~km}$. This means that we can obtain excellent orbital data on fireballs as well as the 


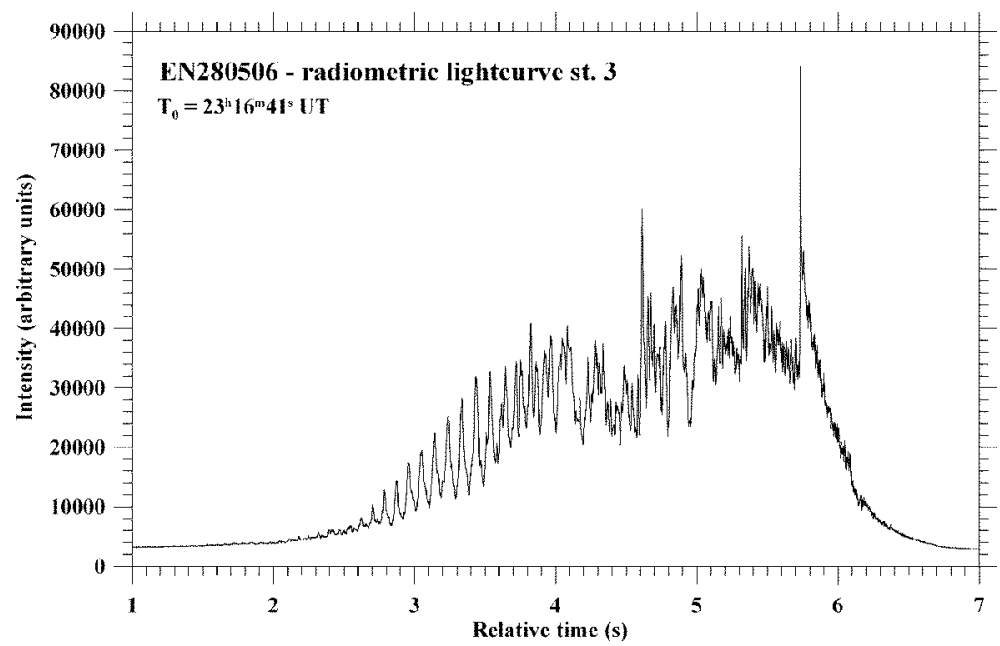

Figure 4. The light curve of the EN280506 as detected by sky brightness sensor installed in the $\mathrm{AFO}$ at Růžová station.

precise positions necessary among others for rapid recovery of meteorites not only from the territory of the Czech Republic but also from surrounding countries.

\section{Results}

We present here complete data on two recent fireballs as an example of the capabilities of our observing system based on AFOs. We show the high precision of all determined values as well as very detailed information about the light curves of both the cases presented.

\subsection{The EN280506 fireball}

This bright and long fireball with a peak absolute magnitude -11.4 was photographed on 28th May 2006 at $23^{h} 16^{m} 42.1^{s}$ UT (the time of the beginning of the photographed trajectory). Photographic records of this fireball were taken on 4 stations of the Czech Fireball Network (see Fig. 3 for the image of this fireball). Autonomous Fireball Observatories recorded this event on stations 10 Polom, 3 Růžová and also at the Ondřejov Observatory, where also fixed and guided manually operated fish-eye cameras were in operation. At station 14 Červená hora the manually operated camera was still in use. It was one of the cases, where the AFOs immediately announced detection of a large event, which enabled us to have first very qualified estimates about the atmospheric trajectory already the second day after the event. Such prompt information is especially important in the cases which could produce a meteorite fall. After the reduction of all available all-sky images, all geophysical, orbital and dynamical data have been computed. The fireball started its almost $110 \mathrm{~km}$ long luminous trajectory at an altitude of $88 \mathrm{~km}$ over the Czech highest mountains Krkonoše with the low entry velocity of $17.6 \mathrm{~km} / \mathrm{s}$ and terminated its flight after 6.8 seconds at a height of $30.5 \mathrm{~km}$ near the Polish town Legnica. The average slope of the atmospheric trajectory to the Earth's surface was 31.3 degrees. Basic results are presented along with the EN300706 fireball in Tables 2 and 3. All values were determined with high precision and well document the capabilities of this observing system.

From its behavior during the atmospheric flight and from its orbit in the Solar system it was a typical asteroidal meteoroid which could produce a significant meteorite if it 

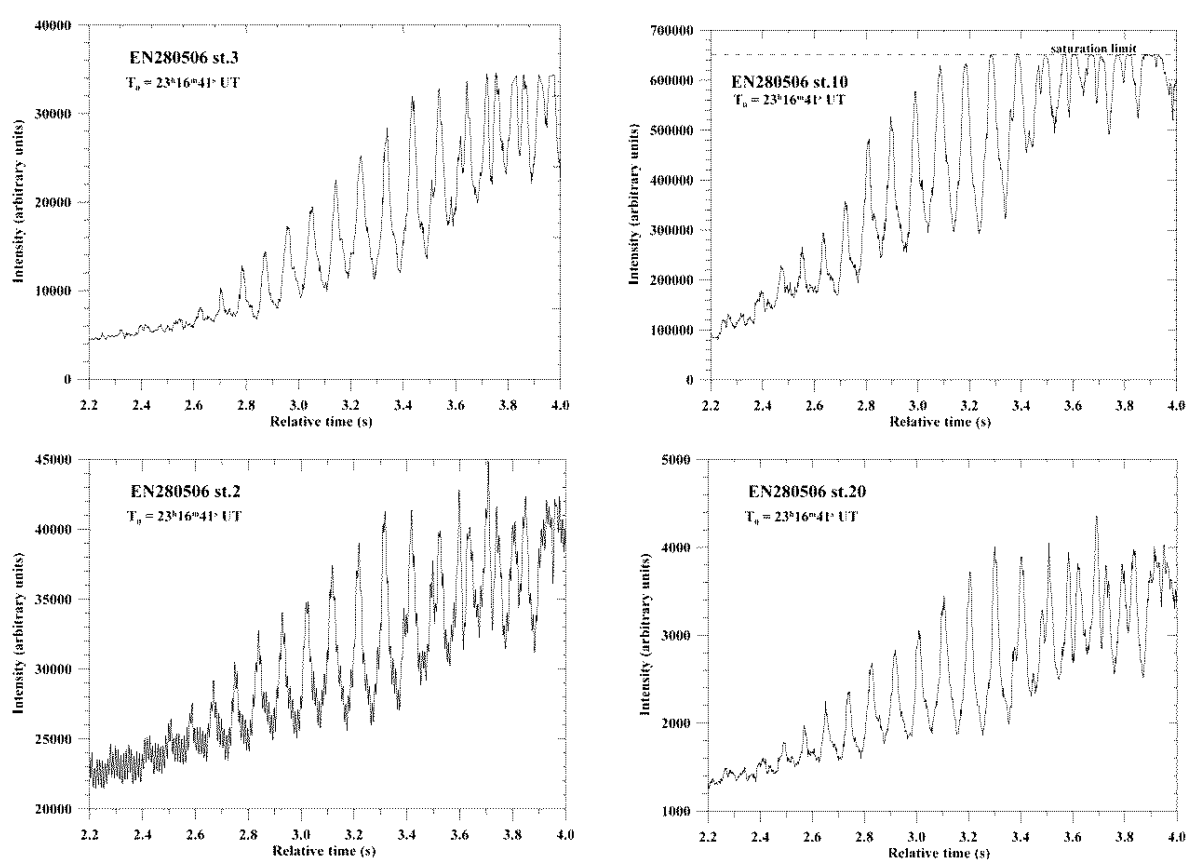

Figure 5. The light curve of the EN280506 fireball as independently seen from four different stations of the Czech Fireball Network. Periodical behavior of the brightness is evident.

would have larger initial mass. In this case the total terminal mass was less than 100 grams which is too low for any systematic search.

The main exceptionality of this fireball consists in its unusual light curve, especially in its beginning part.

The light curve was recorded by the brightness sensors at all AFOs which were that night in the operation. The whole light curve from station 3 Rưžová, which was close to the event but not so close like station 10 Polom where the signal was saturated in the maximum, is shown on the Figure 4. The beginning part of the fireball is enlarged in Figure 5 along with another three stations. Figures 4 and 5 nicely show the high resolution of these records (500 samples/s). From the comparison of independent records from different stations, high fidelity of these records is evident. Even the tiniest details of the light curves are well reproduced. The differences in intensity and noise level are caused only by different distances of individual AFOs from the fireball path. All these details are visible only on these radiometric records and cannot be distinguishable from the photographs where we can observe only some small irregular changes of brightness.

A very interesting and unusual feature is observed in the first half of the light curve. As it is shown in Figure 5, significant periodic fluctuations in brightness are observed shortly after the beginning. There is an easily distinguishable main periodicity, at an almost constant frequency of $10.61 \mathrm{~Hz}$ from the very beginning with increasing amplitude. This effect is not very common. We already observed similar behavior for only one fireball detected by another kind of radiometers with still higher time resolution but lower sensitivity, which is described in Spurný \& Borovička (2001). Such significant periodic variations in brightness could be possibly explained by rotation of highly elongated body before its fragmentation. These records hold unique information about luminous processes during meteoroids atmospheric flight which we have had never before from our observations and therefore the evaluation of these light curves is still not finished 


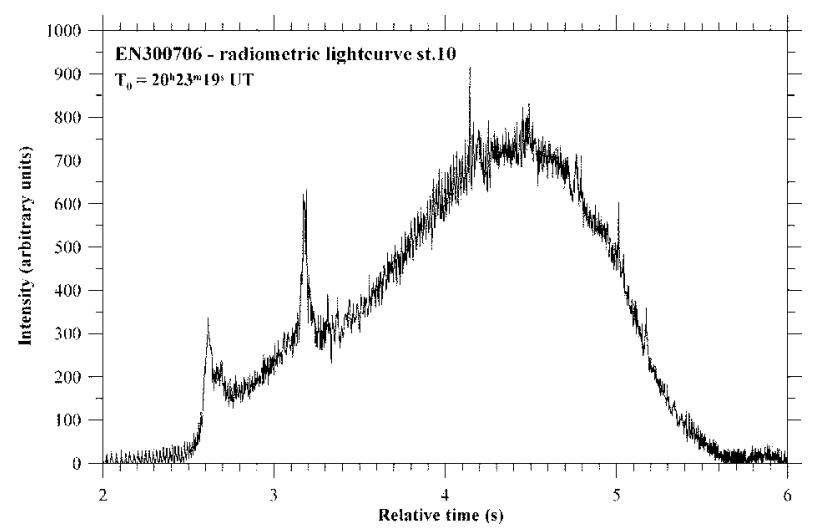

Figure 6. The light curve of the EN300706 as detected by radiometer at Polom station.

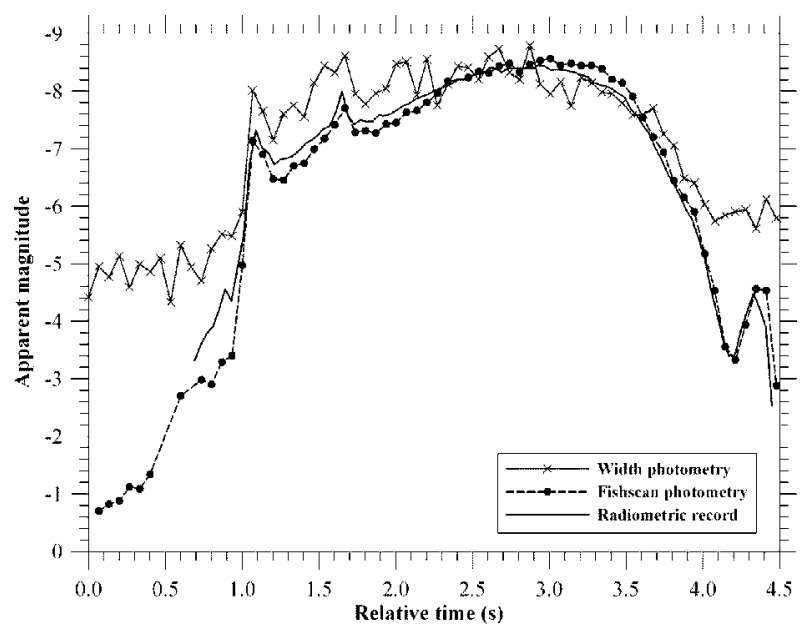

Figure 7. The apparent light curve of the EN 300706 fireball at station 10 Polom as determined by three different methods.

and will be described in more detail in a separate paper. It is presented here mainly for illustration of the capabilities of our new observing instruments.

\subsection{The EN300706 fireball}

The second example of the work of our fireball network is the fireball EN300706. This fireball was not so bright and long as the previous case but it well documents the work of AFOs and also the consistence in results from our new measuring methods, especially the photometry described in Section 3. This fireball was recorded at almost all stations of our network, but the four closest stations equipped only by AFOs were taken for computations. The fireball image taken by the AFO at station 10 Polom is shown in Figure 3. All main results are collected in Tables 2 and 3. This slow moving fireball of -7.3 maximum absolute magnitude started its $68 \mathrm{~km}$ long luminous flight at a height of $83 \mathrm{~km}$ and disappeared after 5 second at a height of $37 \mathrm{~km}$. Like the previous case, this fireball belongs to the type I fireballs and its asteroidal origin is the most likely.

Figure 7 shows a comparison of the fireball light curve at station 10 Polom obtained by three different methods: photographic photometry using trail width measurements by the Ascorecord measuring device, photographic photometry done the scanned image with 
the Fishscan software (described in Sect. 3), and the signal from the photoelectric sky brightness sensor. The sensor data (shown in original form in Fig. 6) have been smoothed to match the temporal resolution of the photograph and transformed to logarithm scale. Since the radiometric signal is not calibrated in magnitudes, the data were shifted vertically to nearly correspond to the photographic light curves at the relative time $2.5 \mathrm{~s}$, near the fireball maximum.

The comparison demonstrates the almost excellent correspondence of the light curve shape between the radiometric and the Fishscan method. Marked differences between both curves can be seen only near the fireball beginning, where, nevertheless, the errors of both methods exceed one magnitude because of the fireball brightness. The sensitivity limit of the radiometer is about magnitude -3 . The Ascorecord curve shows larger scatter and deviates significantly near the fireball beginning and end. The absolute values of the fireball brightness near the fireball maximum, nevertheless, agree well from both photographic methods.

\section{Acknowledgements}

We thank Mrs. J. Keclíková who measured most of the photographic records, and Mr. J. Boček for his maintenance of the fireball stations. This work has been supported by project AV0Z10030501.

\section{References}

Borovička, J. 1990, Bull. Astron. Inst. Czech. 41, 391

Borovička, J., Spurný, P. \& Keclíková, J. 1995, A\&AS 112, 173

Ceplecha, Z. 1987, Bull. Astron. Inst. Czech. 38, 222

Ceplecha, Z., Spurný, P., Borovička, J. \& Keclíková, J. 1993, A\&\&A 279, 615

Spurný, P. \& Borovička, J. 2001, in: B. Warmbein (ed.) Proc. Meteoroids 2001 Conf., ESA-SP 495 , p. 519 